\title{
Surgical Treatment of Left Main Coronary Artery Aneurysm A Case Report
}

\author{
Tzu-Yu Lin, MD*,**; Kuan-Ming Chiu, $\mathrm{MD}^{\dagger} ;$ Jiann-Shing Shieh, $\mathrm{PhD}^{* *}$; Shu-Hsun Chu, MD
}

\begin{abstract}
Left main (LM) coronary artery aneurysm is rare and usually found incidentally during coronary angiography. Except for rare Kawasaki disease, iatrogenic and mycotic aneurysms, atherosclerosis is the primary cause of coronary aneurysm. In most clinical scenarios, coronary artery disease is accompanied with LM coronary aneurysm. Although coronary artery aneurysm does not confer added risk in patients with coexisting obstructive coronary artery disease, LM coronary aneurysm itself remains a significant clinical concern. Thrombosis and distal embolization are the most likely reasons to cause morbidities. Aggressive surgical treatment should be considered. Here, we report a 65-year-old man presenting with effort angina. LM coronary aneurysm was noted in coronary angiogram and images including computed tomography, transesophageal echocardiography and operative photography were presented. (Circ J 2009; 73: 770-771)
\end{abstract}

Key Words: Bypass surgery; Coronary artery aneurysm; Left main; Multi-detector computed tomography; Transesophageal echocardiography

$\mathbf{L}$ eft main (LM) coronary artery aneurysm is rare, with only $0.1 \%$ of adults undergoing coronary angiography! Depending on the clinical scenarios, LM aneurysm can be managed by observation, percutaneous intervention or surgical resection. Atherosclerosis, mucocutaneous lymph node syndrome, autoimmune diseases (systemic lupus erythematosus, periarteritis nodosa or types of arteritis), trauma, dissection, iatrogenic complications (angioplasty, atherectomy, laser procedures), syphilis and mycotic emboli are all possible causes of coronary aneurysms? Surgery should be considered for large LM coronary aneurysms because of the risks of thrombosis and embolism. In fact, aneurysmal coronary artery disease should be considered a variation of coronary artery disease, with which it usually coexists? The coexisting obstructive coronary artery disease plays an important role in making a decision for the operative management in patients with the LM coronary aneurysm.

\section{Case Report}

A 63-year-old man had a coronary stent for left anterior descending (LAD) coronary artery 1 year before and presented with intermittent exertional chest tightness. The treadmill exercise test showed cardiac ischemia. Cardiac catheterization showed LM coronary aneurysm and 2vessel disease (Figure 1A). Multi-slices computed tomography showed significant luminal dilatation of LM coronary artery (Figure 1B). Surgical resection and revascularization

(Received January 31, 2008; revised manuscript received May 23, 2008; accepted May 29, 2008; released online December 15, 2008)

*Department of Anesthesia, Division of Cardiovascular Surgery, Far-Eastern Memorial Hospital, Taipei and **Department of Mechanical Engineering, Yuan-Ze University, Taoyuan, Taiwan

Mailing address: Kuan-Ming Chiu, MD, Division of Cardiovascular Surgery, Far-Eastern Memorial Hospital, 13F, 21, Sec 2, Nan-Ya S Rd, Ban-Ciao, Taipei, 220, Taiwan. E-mail: kmchius@yahoo.com.tw All rights are reserved to the Japanese Circulation Society. For permissions, please e-mail: cj@j-circ.or.jp were proposed. Intraopertive transesophageal echocardiography showed a budding chamber from left coronary sinus, which was compatible with LM coronary aneurysm (Figure 1C). Under cardiopulmonary bypass, coronary artery bypasses of the left internal mammary artery to LAD artery and sequential saphenous vein to ramus intermediate and obtuse marginal branches were constructed in beating-heart manner. The main pulmonary artery was then transected to expose LM coronary aneurysm. The LM coronary aneurysm was carefully dissected (Figure 1D) and totally excised. The other vein graft was used to bypass proximal LAD, ramus intermediate and circumflex arteries (Figure 1E). The resected LM coronary aneurysm showed significant atherosclerosis and concentric vessel wall thickening (Figure 1F). The operation was illustrated in Figure 2. The patient was discharged in good condition.

\section{Discussion}

Coronary artery aneurysms are defined as dilated segments greater than 1.5 times the diameter of adjacent normal coronary arteries? Among them, LM coronary aneurysm accounted for one-fifth of overall incidence6 Most of them are incidental findings during coronary angiography with the intent to search for ischemic heart diseases. Coronary angiography remains the gold standard in the diagnosis of coronary aneurysms. Recent advances of image technologies provide less invasive ways to confirm the diagnosis before an invasive approach. The 64-slices computed tomography provides information regarding the size, shape, location, number and calcification in addition to obstructive coronary artery diseases. Alternatively, giant coronary aneurysms can be detected by transesophageal echocardiography as shown in this patient.

The prognosis of coronary aneurysms is generally favorable. Thromboembolic complications are rare with antiplatelet therapy, and spontaneous rupture generally is rare but occurs more commonly in Kawasaki disease and aneurysms associated with coronary arterio-venous fistula? 


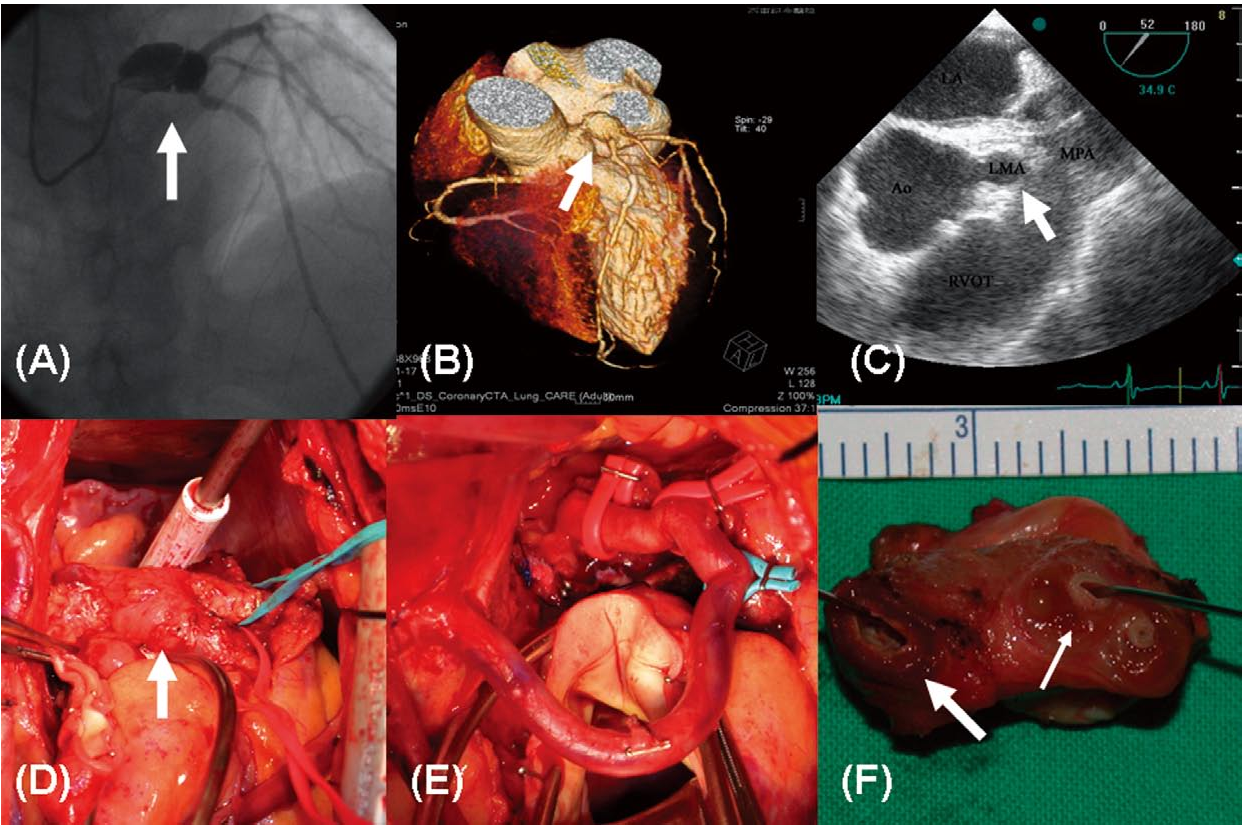

Figure 1. (A) Coronary angiography shows left main (LM) coronary aneurysm and 2-vessel disease. (B) 64-slices computed tomography shows LM coronary aneurysm. (C) Transesophageal echocardiography shows a budding chamber from aortic root. (D) Surgical view of the LM coronary aneurysm. Note the main pulmonary artery was transected. (E) Surgical view after reconstruction. The 3 colored vessel loops indicated proximal left anterior descending (LAD), ramus and circumflex coronary arteries. (F) LM coronary aneurysm specimen. Large arrow indicates LAD and small arrow indicates ramus intermediate orifices. Ao, aorta; RVOT, right ventricular outflow tract; MPA, main pulmonary artery; LA, left atrium; LMA, LM aneurysm.

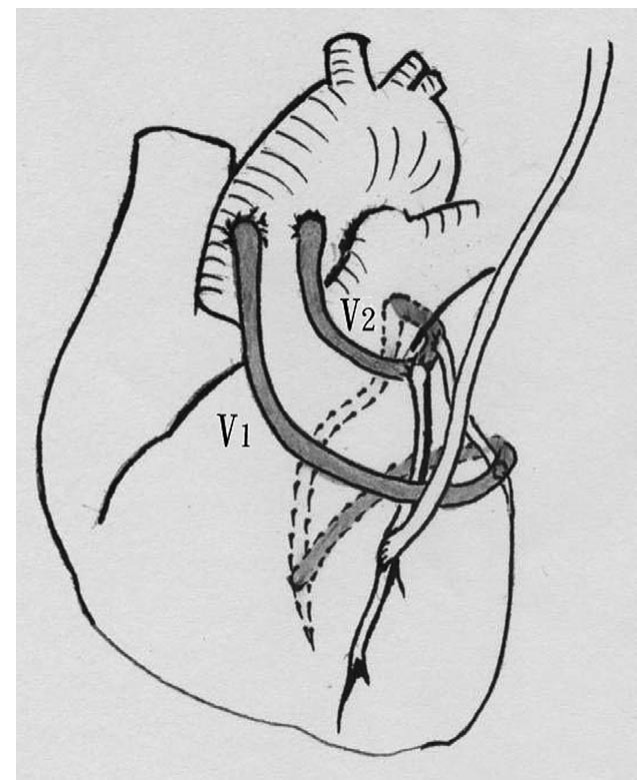

Figure 2. At the first step, off-pump coronary artery bypass surgery was done with the left internal mammary artery to middle left anterior descending (LAD) and the saphenous vein $\left(\mathrm{V}_{1}\right)$ to ramus and obtuse marginal branches. After cardiopulmonary bypass and resection of left main aneurysm, the other vein graft $\left(\mathrm{V}_{2}\right)$ was used to connect the proximal LAD, ramus and circumflex branches.

Although coronary artery aneurysms are mostly asymptomatic and remain benign in clinical behaviors, they might progress to cause thrombosis, distal embolization, rupture and calcification. Operative therapy is usually required for large LM coronary aneurysms because of their predisposi- tion to thrombosis and embolism. This causes large territory of myocardium in risk of infarction. The coexisting significant obstructive coronary artery diseases might be important in making a decision for the operative treatment in patients with the LM coronary aneurysm. Several types of operative approaches had been reported, including patch repair, resection with in-situ grafting and exclusion with distal bypasses.

In our patient, symptomatic, occlusive coronary artery disease is the primary indication for surgical intervention. The nature of atherosclerosis is obvious in extensive aneurysmal remodeling and thickening of vessel wall. Pathology also confirms the diffuse atherosclerotic process in this rare surgical specimen. Excision and bypass grafts exclude all risks related to LM coronary aneurysm. In conclusion, our case provides extraordinary images of this rare clinical entity and also highlights the clinical decision making.

\section{References}

1. Hawkins JW, Vacek JL, Smith GS. Massive aneurysm of the left main coronary artery. Am Heart J 1990; 119: 1406-1408.

2. Park SH, Kim SE, Ryu SK. Left main coronary artery aneurysm with chronic total occlusion of both left coronary arteries in a young athlete. Heart 2001; 85: E1.

3. Demopoulos VP, Olympios CD, Fakiolas CN, Pissimissis EG, Economides NM, Adamopoulou E, et al. The natural history of aneurysmal coronary artery disease. Heart 1997; 78: 136-141.

4. Ercan E, Tengiz I, Yakut N, Gurbuz A. Large atherosclerotic left main coronary aneurysm: A case report and review of literature. Int $J$ Cardiol 2003; 88: 95-98.

5. Burns CA, Cowley MJ, Wechsler AS, Vetrovec GW. Coronary aneurysms: A case report and review. Cathet Cardiovasc Diagn 1992; 27: $106-112$.

6. Harandi S, Johnston SB, Wood RE, Roberts WC. Operative therapy of coronary arterial aneurysm. Am J Cardiol 1999; 83: 1290-1293.

7. Pahlavan PS, Niroomand F. Coronary artery aneurysm: A review. Clin Cardiol 2006; 29: 439-443. 